\title{
THINK TANKS Y SUS ESTRATEGIAS COMUNICATIVAS
}

\author{
José Ramón Santillán Buelna ${ }^{1}$ \\ Universidad Rey Juan Carlos \\ joseramon.santillan@urjc.es
}

Material original autorizado para su primera publicación en la revista académica REDMARKA. Revista Digital de Marketing Aplicado.

https://doi.org/10.17979/redma.2012.01.08.4749

Recibido: 17 Enero 2012

Aceptado 5 Junio 2012

\section{RESUMEN:}

La centralidad comunicativa de la actividad política abre un nuevo horizonte para estudiar los think tanks como promotores y fuentes informativas de los temas políticos, demandas sociales a la búsqueda de respuestas gubernamentales. En este artículo, proponemos la teoría de la tematización para analizar estos centros de estudio y a sus miembros como promotores de estas cuestiones en la prensa. La perspectiva teórica que aquí proponemos considera la importancia de instrumentar una estrategia comunicativa que impulse la difusión del trabajo de investigación de los think tanks y la participación de sus investigadores dentro de las dimensiones mediáticas que caracterizan a la tematización, para impulsar, debatir y buscar soluciones a los temas políticos.

Palabras clave: Think tanks, political issue, tematización, prensa, promotores y fuentes informativos

\footnotetext{
${ }^{1}$ Doctor en Ciencia Política y máster en Teoría Política y Social por la Universidad Pompeu Fabra. Profesor titular interino e integrante del Grupo de Estudios Avanzados en Comunicación (GEAC) de la Universidad Rey Juan Carlos. Ha publicado una decena de artículos en revistas nacionales e internacionales centrados en el campo de la comunicación política
} 


\section{ABSTRACT}

THINK TANKS AND THEIR COMMUNICATION STRATEGIES

The communication-centric approach of political activity opens new horizons to study the role of think tanks as advocates of political issues, of controversial topics to the search for solutions, and sources of information about them. In this article, we suggest thematisation theory to analyse these centres and their members to learn both how they communicate the results of their research, and how they prop up the debate of political topics in the press. To adapt the theoretical perspective we hereby propose, we bear in mind the importance of implementing a communication strategy to foster awareness of the think tanks' work and participation of their researchers in the information dimensions offered by the political issues' newsworthiness to the political success.

Keywords: Think tanks, political issue, journalistic thematisation, information drivers and sources. 


\section{INTRODUCCIÓN}

La formación de la esfera pública es uno de los aspectos más interesantes que ha centrado el estudio de la comunicación política para investigar el papel de los medios en su función de establecer los temas de la agenda de discusión pública. Por ello, un marco para el análisis de la participación de los actores sociales en la vida pública pasa por investigar la gestión de sus demandas, que implica la visibilidad mediática para influir en las soluciones, como un componente básico de la centralidad comunicativa que tiene la política.

La necesidad de gestionar la visibilidad de las demandas sociales en la esfera pública mediática (Thompson, 1995) ha llevado a todo tipo de actores sociales a crear o contratar think tanks, laboratorios de ideas o centros de expertosi, cuyo objetivo es estudiar los problemas sociales para proponer soluciones (Castillo, 2011:50). En ese sentido, resulta de especial interés la comunicación de los resultados de las investigaciones a las audiencias implicadas con el objetivo de influir en la producción de contenidos informativos para condicionar la actividad política. De hecho, estas instituciones y su función investigadora facilitan la participación de determinados grupos en la formación de los temas de la agenda pública que buscan una respuesta política.

En la actualidad, estamos presenciando la fundación de think tanks en todas las esferas de la actividad social (Tello, 2008). Por ejemplo, en la política diseñan y evalúan a la administración pública, o fomentan la ideología de los partidos políticos. En la economía, producen estudios para la toma de decisiones. En la sociedad, promueven ideas como el respeto al medio ambiente. El resultado es que cada vez tienen más influencia como promotores de los problemas públicos en la prensa, lo que ha puesto de relieve su estudio dentro del marco teórico de la tematizaciónii, entendida como el análisis profundo de los temas de discusión que concentran la atención pública y movilizan a determinados grupos a imponer soluciones políticas. 
Es así que para estudiar los think tanks como promotores y fuentes informativos de los asuntos públicos en la prensa tomamos como punto de partida la teoría de la tematización propuesta por Niklas Luhmann (1978) y posteriormente desarrollada por los autores italianos Grossi y Marletti. Este enfoque nos servirá para analizar en la prensa la importancia de comunicar a las diferentes audiencias los resultados de las investigaciones de los centros de expertos como instrumento para impulsar, debatir y buscar soluciones a los temas políticos.

De esta manera, consideramos los think tanks en tanto instituciones que desarrollan estudios sobre la dinámica social, que luego servirán para que determinadas organizaciones gestionen la comunicación de sus demandas con el objetivo de influir en las resoluciones políticas. Se trata de analizar el papel que tienen estos centros y sus miembros como actores en la tematización en la prensa. Un espacio donde se promocionan los problemas sociales y que representa una oportunidad para identificar los criterios de producción informativa que se aplican los periódicos al seguimiento de estas cuestiones.

Para estudiar los think tanks y sus estrategias comunicativas, comenzaré señalando el enfoque básico de la tematización para el estudio de los temas políticos. Después, definiré los think tanks, sus actividades de investigación, funciones y su papel como fuentes y promotores informativos de los temas políticos. Por último, interpretaré los instrumentos de comunicación que utilizan y la labor de los investigadores como articulistas en el ámbito de la tematización. Este análisis parte de las siguientes preguntas: ¿cómo las demandas sociales logran proyección mediática? En este proceso, ¿cuál es el papel de los think tanks y de sus miembros?, ¿cómo y en qué momento participan en la tematización?, ¿qué instrumentos de comunicación utilizan y con qué objetivos?

\section{Tematización: un marco para el estudio de las demandas sociales}


Partimos de que como resultado de la centralidad informativa en la actividad política en las sociedades modernas (Grossi, 1985:14), una característica principal del estudio de la comunicación política se centra en las relaciones entre la estructura de medios y el sistema político como actores en el establecimiento de la opinión pública. En ese contexto, la aportación teórica de la tematización, formulada por Niklas Luhmann en su texto Offentliche meinung en la década de 1970, entendida como el proceso de definición y debate público de los temas políticos determinados por los media (Saperas, 1987:92), abrió una nueva línea para estudiar las demandas sociales donde los medios de comunicación son piezas fundamentales para el equilibrio y el sistema social. Proponiendo que la importancia de los media está en la capacidad de construir la opinión pública, al determinar la orientación del interés ciudadano sobre un conjunto de temas que reclamen el debate público. Este hecho lleva a Luhmann a considerar a la opinión pública como una estructura temática de la comunicación política, una configuración común de sentido que permite el reconocimiento de unos temas como interés general. El hecho de que tales asuntos sean reconocidos por todos como importantes y de que además todos -potencialmente todos- sepan a qué se refieren, aunque la concreción de opiniones discrepen, eso es la opinión pública.

De esta forma la tematización está generada por la comunicación pública desarrollada por el sistema político, donde los medios funcionan como instituciones destinadas a organizar a la opinión pública, y actuarán como mecanismos reductores de la actividad política al establecer los temas de la agenda de discusión.

Este marco teórico sirvió para que autores italianos como Franco Rositi, Giorgio Grossi y Carlo Marletti, entre otros, desarrollaran en la función de tematización un modelo conceptual para explicar cómo se forman y deliberan las demandas sociales que los medios seleccionan para convertirlas en temas políticos, demandas sociales que son expuestas públicamente para la búsqueda de soluciones. El análisis de estas controversias en cuanto a su producción informativa, noticias y opiniones, explicará la función que realizan los medios 
para seleccionar, discutir y solucionar las reclamaciones sociales. Esta propuesta de estudio examina los temas políticos y su tratamiento informativo como base conceptual para el análisis de la difusión investigadora de los think tanks que busca impulsar temas políticos.

\subsection{Definición y características de los temas políticos}

El estudio de la tematización se centra en reconocer y analizar los temas políticos seleccionados por los medios de comunicación en función de las necesidades del sistema político. El tema político se define como una demanda pública que implica un conflicto social, que es presentado y discutido en los medios de comunicación para tomar soluciones políticas. Estas cuestiones exigen un nivel de enfrentamiento social que conduzca a una discusión pública entre los actores políticos involucrados para la aplicación de respuestas, traducidas, generalmente, en su incorporación a la agenda de gobierno (Marletti, 1985: 22-25). Por lo tanto, el tema tiene su base en el ámbito político y tiende a redefinir el marco de las interacciones entre los grupos sociales.

Los temas políticos se originan en el proceso de disputa política entre los actores, y su entrada en la agenda de gobierno pasa por el reconocimiento público de su relevancia y, seguidamente, por generar una dinámica de tomas de posición en los ambientes políticos y sociales influyentes que conduce a un debate para solucionarlos (Badia, 1991:202). Esta concepción de tema político está enmarcada en la transformación estructural del sistema político, debido al creciente predominio de las controversias públicas como orientadores para las decisiones del gobierno, así como al funcionamiento de las lógicas de consenso en las sociedades contemporáneas y los criterios de la regulación de la competencia política entre los actores. Por ello, en la actualidad los conflictos sociales se han convertido en recursos estratégicos de la política, tal cuestión supone: "la capacidad de imponer, guiar, articular, los temas/issues en función de la acción política que ha habido (o bien integrarse) al papel de los medios en este terreno" (Badia, 1991:172). 
La definición de tema político también se sostiene en el concepto de opinión pública planteado por Niklas Luhmann (1989), que la considera una estructura selectiva de temas de la comunicación política. El académico alemán le asigna la función de instrumento guía del sistema político que reduce la elevada contingencia de eso que es políticamente posible, por la vía estratégica de adaptar la organización temática de los procesos de comunicación pública a las constantes demandas de la sociedad y a las respuestas del gobierno. Es así que esta selección de temas responde a la necesidad de encauzar las demandas sociales que de no atenderse desbordarían el control y la respuesta política, porque vivimos en una sociedad llena de actores que reclaman atención y respuesta a sus peticiones. Se trata de asuntos sociales como: generar empleo, desarrollo de servicios públicos o de cuestiones políticas (elecciones, reformas, representación partidista), que activan la negociación política.

La selección de temas políticos tiene consecuencias directas. En el ámbito político, para los actores involucrados se abre un procedimiento para valorar su importancia y una oportunidad política para definir qué posición deben asumir sobre el tema y el grupo que lo promueve. Tanto si es de apoyo como de rechazo participarán en el debate público para construir un marco de argumentación acorde a su postura. De forma paralela, en el ámbito de los medios de comunicación, aparecen una serie de actores tanto del sistema político: miembros de los partidos políticos, funcionarios, candidatos electorales; como de miembros del sistema mediático: periodistas, articulistas; y también de la comunidad social y cultural como académicos, investigadores, intelectuales que con sus colaboraciones en prensa configuran y determinan la discusión de la demanda en controversia (Santillán, 2007:26-28).

Los temas políticos generan que el gobierno y los partidos políticos se esfuercen en solucionarlos. También involucran a los medios de comunicación, que serán los instrumentos para plantear la discusión pública, a través de una fuerte participación, no sólo para apoyar o rechazar posiciones, sino para construir ellos mismos una propia. 
Para analizar la importancia política de las demandas sociales, el investigador italiano Marletti (1985:65) señala tres etapas que resumimos en el cuadro 1. Primero, su selección; segundo, su discusión y tercero, las soluciones. En la primera fase predomina el conflicto para convertirse en asunto de interés político e informativo. Su importancia está determinada por el grado de controversia social y su prioridad política, que luego dará paso a la institucionalización del tema, momento en el que los grupos políticos decidan implicarse. En este ciclo se analiza a los problemas sociales y los actores para explicar su preferencia a determinadas cuestiones.

Cuadro 1. Características de un tema político y el análisis de cada etapa.

\begin{tabular}{|c|c|c|}
\hline Etapa & Características & Análisis \\
\hline Selección & $\begin{array}{l}\text { - Importancia del } \\
\text { conflicto } \\
\text { - Oportunidad política } \\
\text { - Institucionalización }\end{array}$ & $\begin{array}{l}\text { - Examina a los problemas sociales y } \\
\text { los actores para explicar la elección } \\
\text { política a determinados temas. }\end{array}$ \\
\hline $\begin{array}{l}\text { Discusión/ } \\
\text { Argumentación }\end{array}$ & $\begin{array}{l}\text { - Señalar causas } \\
\text { - Actores implicados } \\
\text { - Posturas } \\
\text { - Alternativas de } \\
\text { solución }\end{array}$ & $\begin{array}{l}\text { Se identifica en el discurso } \\
\text { periodístico a los actores implicados } \\
\text { y sus estrategias argumentativas. } \\
\text { Así como las decisiones políticas }\end{array}$ \\
\hline Solución & - Respuestas políticas & \\
\hline
\end{tabular}

La segunda fase es el proceso de discusión o argumentación, que consiste en conocer las posturas políticas. Y la tercera es la implementación de soluciones, que se traduce en un nuevo equilibrio del sistema político. En estas dos etapas se estudia el discurso periodístico para identificar a los actores implicados en su esfuerzo por desarrollar estrategias argumentativas que garanticen el apoyo a sus intereses. Por lo tanto, consiste en investigar los marcos argumentativos 
del tema: las causas, la oportunidad para resolverlo, los actores implicados y sus posturas, las alternativas de solución y las decisiones políticas tomadas. Estos dos niveles nos llevan a examinar la información publicada en medios de referencia porque se busca conocer la influencia de los grupos y de la prensa en los ámbitos de decisión que negociarán soluciones del tema en discusión.

\subsection{Temas políticos y su discurso periodístico}

El modelo de tema político aquí desarrollado concede gran importancia a la actuación de los medios de comunicación. La forma en que se seleccionan y discuten los temas es un factor clave para resolverlos. Los medios contribuyen a que determinados grupos puedan plantear un tema y, por tanto, una respuesta. Estos asuntos que generan controversia política son conocidos por los ciudadanos y reciben un tratamiento informativo. La exposición en los diferentes medios da paso a la tematización, que consiste en organizar el debate, formar opiniones y discutir en profundidad los problemas para alcanzar consensos elementales. Este escenario permite estudiar el discurso periodístico no sólo como una realidad noticiosa, sino como un espacio de interacción entre múltiples actores sociales en torno a la promoción y respuesta política de un conflicto social.

Para explicar el tratamiento periodístico de un tema político, se parte del estudio de las rutinas informativas que consiste no sólo en la capacidad de seleccionar el tema, sino en una competencia de contextualización, entendida como un análisis profundo de los argumentos con que se debate en los medios. Desde esta perspectiva Rositi (1982:138-139) señala que el examen tiene dos fases. La primera es la selección del tema que consiste en: información primera (noticias), información tematizada (editorial), fuentes y promotores informativos. La fase dos es la contextualización política que está formada por: la definición del tema, causas, responsables y soluciones.

La primera fase comienza con la selección del tema que pone en marcha la producción informativa. Su controversia política da derecho a acceder al circuito informativo -referencias en las noticias-. Una vez que el asunto es 
conocido se inicia la discusión para reflexionar sobre él y debatirlo con los demás, que se manifiesta en las posturas desarrolladas en la producción editorial información segunda o tematizada (Rositi, 1982:138-139).

La selección de temas políticos, por su importancia social, centran el interés de los medios (Raboy y Dagenáis, 1992:3) que serán inmediatamente recogidos en noticia, y después en forma de opinión. Estos dos tipos de información también se dirigen a dos audiencias distintas: la del conjunto de todos los ciudadanos y la de los actores afectados. En el primer caso el mensaje es general dirigido a los diferentes públicos que conocen el tema a través de las noticias. En el segundo grupo ya es especializada, se presenta en forma de editoriales y artículos donde los actores implicados actúan en el terreno de la interpretación para explicar su posición ante los grupos decisorios y lograr el respaldo a su demanda. Esta batalla se da en las páginas de la prensa de referencia porque es el ámbito donde se localizan los grupos de decisión (Borrat, 1998:21). La información noticiosa tiene como objetivo que el tema político reciba cobertura mediática. En cambio, la tematización que la producción de opinión nutra la discusión entre los actores involucrados con la finalidad de influir en las soluciones. De esta manera, el periódico tiene influencia política porque se convierte en el espacio de la lucha pública, en donde los protagonistas sociales expresan sus puntos de vista.

La segunda fase del análisis de la tematización periodística es su contextualización, que da inicio a la discusión pública del tema político. Los editoriales y artículos son ejemplo de relato periodístico que exponen el origen del tema, señalan responsabilidades políticas y desarrollan soluciones. A medida que se explica, un tema político pasa por la argumentación de una gran cantidad de articulistas con acceso a la prensa, produciéndose así una diversidad de opiniones cuyas interpretaciones varían y se retroalimentan unas a otras. Los temas políticos son material editorial permanente en el sentido de que están constituidos por una pluralidad de razonamientos que se producen a medida que se expone. Los participantes en la disputa dan a conocer sus 
posturas en estos espacios donde se revelan sus vínculos políticos. El debate crea un ámbito donde se confrontan cada día al hilo de la publicación de artículos con nuevas interpretaciones y opiniones.

En este trabajo para el estudio de un tema político en la prensa nos centraremos en el análisis de quiénes participan en su promoción y debate en los medios de comunicación, ya que como observa Gusfeld "la arena política no es un campo de juego en que juegan todos bajo las mismas reglas; algunos tienen mayor acceso que otros y también detentan un poder mayor para dar forma a la definición y debate de los problemas públicos" (Gusfeld,1981:23). Por sus características de fenómenos políticos con una alta actividad mediática, los temas políticos muestran la participación de una gran cantidad de personas de distintos ámbitos sociales. En ese contexto, el análisis de los de los centros de estudio y de sus integrantes como fuentes informativas consistirá en estudiar su papel en la promoción de demandas sociales, considerando que su importancia como productores de información se debe a la calidad de sus investigaciones y por al alto reconocimiento profesional que les brinda un acceso directo a los medios de comunicación. Su participación como expertos en la prensa de referencia jugará un papel importante en la discusión o argumentación política del tema. Con estas claves analizaremos la difusión de la actividad investigadora para reconocer su capacidad de promover problemas sociales, que explicará cómo determinados actores e instituciones tienen mayores recursos para influir en la agenda de temas y en las decisiones políticas. El cuadro 2 muestra los puntos del análisis de la tematización, que nos servirán para estudiar las estrategias argumentativas de los think tanks en la prensa.

Cuadro 2. Fases y análisis de la tematización en la prensa. 


\begin{tabular}{|l|l|l|}
\hline \multicolumn{1}{|c|}{ Fase } & \multicolumn{1}{|c|}{ Características } & \multicolumn{1}{c|}{ Análisis } \\
\hline Selección & $\begin{array}{l}\text { Información primera } \\
\text { (noticias) } \\
\text { Información tematizada } \\
\text { (producción editorial) }\end{array}$ & $\begin{array}{l}\text {-Medición de la visibilidad del } \\
\text { tema, se cuentan titulares y todas } \\
\text { las noticias. }\end{array}$ \\
& $\begin{array}{l}\text {-Medición del inicio de la } \\
\text { tematización, se cuenta los } \\
\text { artículos publicados. }\end{array}$ \\
& $\begin{array}{l}\text { informativas } \\
\text {-Identificación de los actores que } \\
\text { promueven noticias o producen } \\
\text { artículos del tema. }\end{array}$ \\
\hline Contextualización & $\begin{array}{l}\text { Definición del tema } \\
\text { Protagonistas } \\
\text { Causas y responsables }\end{array}$ & $\begin{array}{l}\text { - Identificar en el discurso } \\
\text { periodístico a los actores } \\
\text { implicados y sus argumentos. Así } \\
\text { como las decisiones políticas } \\
\text { sugeridas }\end{array}$ \\
\hline
\end{tabular}

\section{Think tanks como promotores y fuentes informativas de temas políticos}

En este apartado analizamos los think tanks en cuanto a sus actividades de estudio para conocer su rol como promotores e identificar la participación de sus investigadores como fuentes informativas de los political issues. De esta forma los definimos, señalamos los tipos que existen, sus funciones y por último, ilustramos la comunicación de resultados de su trabajo investigador para comprender su papel como impulsores de temas políticos en los medios.

\subsection{Definición y tipos}

En la actualidad, los think tank concepto que significa laboratorio de ideas 0 centro de expertos (Castillo, 2009), son organizaciones en alza que brindan información a diferentes grupos sociales con el objetivo de que la proyección pública de sus demandas se sitúe en el centro de la atención comunicativa y el interés político. Desde este punto de vista existen diversos modelos de centros de estudio con una estructura, funciones y actividades diferenciados entre sí, 
pero con el objetivo común de apoyar con conocimiento experto a los intereses de los diferentes grupos para influir en el debate político e informativo. Ferran Requejo y su grupo de investigadores de la Universidad Pompeu Fabra (1999; 46-47), describen seis tipos teniendo en cuenta su organización: (1) centros universitarios de investigación (universities without students), formados por profesores universitarios que comparten su actividad docente con la investigación básica y la producción de conocimiento práctico; (2) centros de investigación vinculados a la administración (In house service), instituciones creadas y financiadas por el gobierno para realizar, planificar y evaluar políticas públicas. También, promueven el análisis y debate de temas de interés público; (3) centros de investigación vinculados a los grupos de interés, son instituciones de estudios para apoyar los intereses políticos, económicos y sociales de determinados grupos; (4) organización sin ánimo de lucro. Grupo de personas que promueven ideas y valores sociales; (5) centros identificados a partidos políticos (advocacy tanks), promueven su ideario en el debate político; (6) consultores privados, profesionales que trabajan en agencias de comunicación y de relaciones públicas.

La particularidad de investigación realizada por un think tank requiere de personas con unas características determinadas que se enmarcan en una alta formación universitaria interdisciplinaria, deben dominar diversas áreas de conocimiento para poder analizar los fenómenos sociales, y manejar adecuadamente los métodos de investigación. También, es necesaria una habilidad para identificar actores y posibles cursos de acción y entender el funcionamiento de los medios de comunicación para poder comunicar su trabajo. La reputación académica es la seña más representativa de los miembros de los centros de estudio.

Estas cualidades de sus investigadores y la especificidad de la labor de investigación de cada think tank los convierte en actores que les permite funciones específicas de influencia política que a continuación desarrollamos. 


\subsection{Funciones}

La investigación académica sobre los think tanks (Wallace: 1994, 142-143) les confiere múltiples funciones de entre las que destacamos dos. Por un lado, el análisis de los problemas sociales y, por otro lado, su capacidad para apoyar la participación de grupos organizados en el proceso de decisión política. Ambas tienen un componente político y comunicativo importante (Xifra, J. 2005.:75), ya que el análisis de los asuntos sociales requiere estudios que son usados como información de base para la gestión política que se hará pública mediante la implementación de estrategias comunicativas. Por lo que respecta a la participación de grupos organizados, el conocimiento en torno a sus demandas con el uso de los medios de comunicación resultará clave para que sean objeto de tematización.

Un think tank realiza una serie de análisis de la actividad política para determinados actores e instituciones y los resultados de su trabajo servirán para diseñar estrategias comunicativas con el objetivo de fijar el problema ante la opinión pública y movilizar a los decisores políticos. En este marco la tematización que se alimenta de artículos y editoriales se ha revelado como una formidable herramienta de debate y negociación de los temas políticos (Santillán, 2009:126-127). Por lo tanto, los actores que buscan respuestas políticas a sus demandas se apoyan en los resultados de las investigaciones de los grupos de estudio para formular una estrategia de comunicación en la prensa.

2.3 La difusión de resultados de investigación como estrategia comunicativa.

La estrategia comunicativa de los problemas públicos consiste en primer lugar, en que el tema centre la atención informativa y posteriormente, sea objeto de tematización. En este escenario, las conclusiones de los trabajos de los think tanks son un importante material informativo para impulsar demandas en la esfera mediática que nutren las líneas de acción política que comienza con exponer el problema, los personajes, sus causas y las soluciones. Así, la 
comunicación es importante para impulsar demandas sociales que centrarán la agenda de discusión pública.

En segundo lugar, otro ámbito del diseño de estrategias comunicativas con información proveniente de los think tanks tiene como objetivo influir en los grupos de decisión. En está situación las organizaciones necesitan construir un clima de opinión favorable entre los actores sociales que tienen poder de resolución política. Para influenciar a estas audiencias fomentan que los integrantes de los centros de investigación participen como articulistas en la prensa. Los especialistas en sus textos intentan persuadir al lector para que tome una postura en función de los argumentos que ellos desarrollan. En este escenario, los expertos como comentaristas son pieza clave en el debate porque no sólo refuerzan la capacidad de la prensa para decirnos en qué pensar, sino con qué palabras. La importancia que el lector otorga a los artículos de los investigadores se basa en su reconocimiento y prestigio social, y si consideramos que los integrantes de los centros de investigación ejercen su actuación en los espacios de opinión de diarios de prestigio, tienen impacto para influir en los grupos de decisión. Todo esto nos permite afirmar que la difusión de los resultados del trabajo de los think tanks y el rol como articulistas de sus miembros son importantes instrumentos para que los grupos implicados gestionen de forma eficaz sus demandas. Produzcan, controlen y dirijan información esencial a los diversos públicos. Por lo tanto, la buena comunicación de los problemas sociales se convierte en una estrategia decisiva para impulsar temas con el objetivo de intervenir en las decisiones políticas.

La importancia de influir políticamente en los grupos decisorios hace que los media sean un elemento relevante conectado al trabajo de los think tanks. En este proceso de influencia la forma en cómo los grupos sociales difundan los estudios elaborados por los especialistas, será factor importante para que los políticos atiendan sus demandas. De manera particular, en la tematización en prensa de temas políticos informar a las audiencias clave será decisivo para 
organizar el debate, formar opiniones y discutir en profundidad para alcanzar consensos.

2.4 Think tanks y sus investigadores como promotores de temas políticos

Para ilustrar el trabajo de los think tanks y de sus miembros como promotores de temas políticos, se propone el cuadro 3 que continuación explicamos:

Cuadro 3. Think tanks como promotores de temas políticos

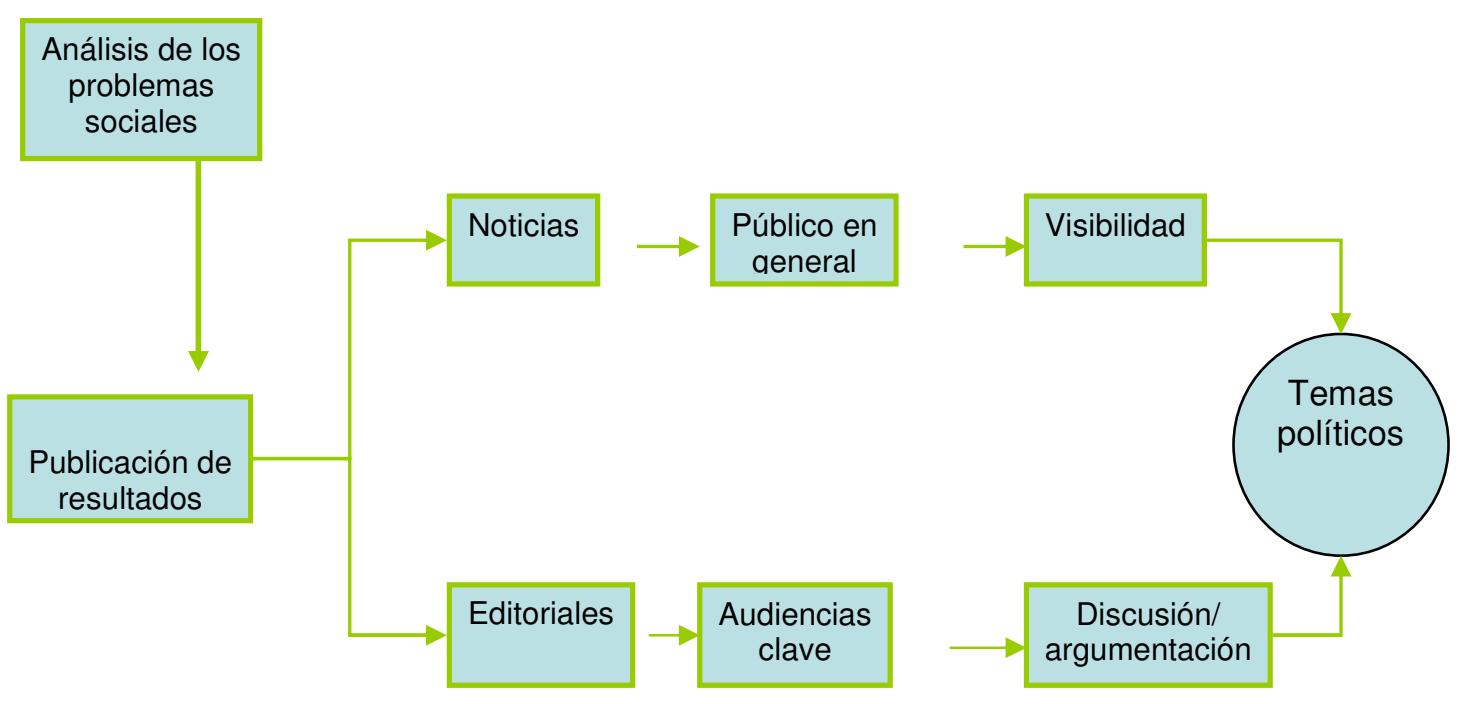

Los centros de expertos desarrollan estudios de los problemas sociales para apoyar el logro de objetivos de los grupos que los contratan. Con los resultados de sus investigaciones se elaboran estrategias comunicativas para promover nuevas demandas con dos fines: que sean visibles y desaten una discusión mediática. Los mensajes se transmiten en dos dimensiones: al público en general y a los grupos de decisión con el objetivo de informar y después editorializar. En el primer caso, la información es genérica dirigida a una audiencia que conoce el tema político a través de las noticias en todos los medios de comunicación. En el segundo grupo es editorial los actores involucrados se enfrentan en el terreno de la interpretación para explicar a los grupos de decisión su postura y lograr apoyos. Como explicamos anteriormente esta batalla se da en los artículos de opinión de la prensa de referencia porque es el ámbito en donde se localizan estos grupos. En la promoción de temas políticos contar con una estrategia comunicativa es muy importante para 
difundir el trabajo de los think tanks en los medios de comunicación, para que los políticos atiendan sus demandas y ejecuten las soluciones. En el centro del debate sobre cómo hacer públicas estas demandas está una buena gestión informativa. Transmitir los resultados del análisis de los problemas sociales es estratégico porque va más allá de las noticias, pues implica información editorial sostenida en análisis críticos que contempla toda la complejidad de la exigencia para influir en las personas implicadas en tomar decisiones políticas.

Por todo ello, los centros de investigación y los medios de comunicación constituyen un binomio imprescindible. Planteamos el think tank como un organismo que puede ser analizado desde el campo de la información noticiosa y editorial, que primero visibiliza y luego promueve la discusión del tema político con el objetivo de convencer a los decisores políticos. Así, los investigadores y su dinámica comunicativa que a continuación analizaremos, señalan cómo para determinados grupos sociales la publicación de los resultados de su trabajo facilita el impulso de las demandas sociales. Bajo este punto de vista, es en los medios de comunicación donde se proyectan dichas cuestiones, que se inicia con las noticias, construyendo un conocimiento público del tema en el ciudadano, y continúa en los artículos de opinión, como estrategia discursiva en la tematización en la prensa.

\section{Think tanks e investigadores en la discusión periodística.}

Para participar en la tematización y relacionarse con los actores durante un tema político, los think tanks utilizan diversos instrumentos de comunicación para lograr determinados objetivos ver cuadro 4. Xifrau (2005) destaca entre los canales habituales para la difusión de la actividad investigadora: ruedas de prensa, la participación en foros, congresos, seminarios, presentación de libros, conferencias, publicación de artículos y entrevistas. Estas tareas sirven para promover directamente asuntos públicos que garantizarán una cobertura informativa, debido al prestigio que tienen los centros de expertos y al reconocimiento profesional de sus miembros. 
En la tematización, informar sobre las demandas es el primer objetivo para que la selección del tema por los medios de comunicación que proyectarán intereses, interlocutores y escenarios donde se dará la disputa política. En este ciclo la dinámica del conflicto se nutre con noticias de diversos instrumentos de comunicación que tiene el think tank como son las ruedas de prensa, que comprende difundir los resultados de las investigaciones con el fin de lograr titulares y presencia mediática del tema.

Cuadro 4. Instrumentos de comunicación de los think tanks en la tematización.

\begin{tabular}{|l|l|l|}
\hline Instrumentos de comunicación & \multicolumn{1}{|c|}{ Objetivo } & Tematización \\
\hline - Comunicados & $\begin{array}{l}\text { Proyección pública de } \\
\text { demandas, interlocutores y } \\
\text { escenarios donde se dará la } \\
\text { - Presentación de libros }\end{array}$ & Selección del tema \\
- Informes de investigación & $\begin{array}{l}\text { dispolítica } \\
\text { - Entrevistas }\end{array}$ & $\begin{array}{l}\text { - Encuadrar la problemática } \\
\text { del tema e iniciar la formación } \\
\text { de opinión }\end{array}$ \\
\hline - Los investigadores comolistas & $\begin{array}{l}\text { Darticipar en el debate para } \\
\text { intervenir en las soluciones }\end{array}$ & \\
\hline
\end{tabular}

Pero la difusión de resultados del trabajo de los think tanks no sólo busca recibir cobertura informativa, sino participar en el debate y solución política del asunto en conflicto. Para lograr este objetivo sus investigadores colaboran en prensa como articulistas con la que se inicia la tematización. En esta etapa los expertos se implican en definir los problemas y debatirlos con los demás para resolverlos. El objetivo es influir en los grupos que decidirán y es en los medios de referencia donde despliegan su propósito de persuasión. La influencia 
política viene dada por que un artículo en un diario de prestigio tiene mayor impacto para conseguir apoyo en los centros de poder.

La participación de los investigadores dentro de la tematización tiene la función de interpretar el tema en conflicto para persuadir al público para que tome posición en función de los argumentos que ellos desarrollan.

Por todo lo anterior, think tanks e investigadores actúan como promotores y fuentes informativas que definen la importancia del tema, señalan causas, actores implicados y proponen respuestas políticas. Cada uno de estos aspectos les ofrece la posibilidad de participar en la producción periodística. Para los expertos su papel como articulistas implica análisis y alto conocimiento de la demanda y un acceso directo a los medios de comunicación que no todos los participantes tienen. Se trata de contribuir en la discusión generando opinión, apoyo o rechazo de posturas e intervenir en las soluciones.

Para ello los grupos aprovechan a los miembros de los think tanks como productores de contenidos editoriales para participar en los espacios de opinión, ámbito donde se discuten y se sugieren soluciones de los asuntos que componen el debate político. En este entorno, los investigadores como articulistas participan activamente en la tematización al promover demandas concretas de determinados grupos sociales. Este influjo descansa en el prestigio profesional del investigador que con sus colaboraciones en prensa de referencia, un medio muy selectivo, le permitirá llegar a los grupos con gran poder de decisión política.

La visibilidad mediática de los think tanks y de sus miembros los erige en promotores de los problemas públicos, practicando la tematización, que para los grupos sociales es un elemento estratégico para el éxito de sus intereses. De ahí que es importante considerar la función y estrategias argumentativas de los artículos de opinión en la prensa como instrumentos para influir en las decisiones políticas. 


\section{Conclusiones}

Enmarcar los think tanks y a sus investigadores dentro del enfoque de la tematización y adaptar este modelo para analizarlos en la dinámica de un tema político, es reconocer su importancia como actores comunicativos. En este escenario, se resalta su papel para impulsar y debatir las demandas sociales dentro de la centralidad que la comunicación tiene en el desarrollo de la actividad política. Además, su estudio como fuentes y promotores informativos de los temas políticos en la prensa supone concebirlos como protagonistas en la construcción de la esfera pública.

Como hemos visto en este artículo, para los grupos sociales la tematización es una pieza fundamental para el logro de sus intereses políticos. Desde el punto de vista de los think tanks está relacionada con su función investigadora de los problemas sociales cuyos resultados de trabajo se comunicarán a todas aquellas personas implicadas en las decisiones políticas. Desde la perspectiva de sus expertos implica la posibilidad de actuar directamente como comentaristas a través de su participación como articulistas.

Por lo tanto, cualquier organización que busca soluciones políticas a sus demandas necesita considerar el diseño de estrategias comunicativas para movilizar a determinados grupos y lograr su apoyo. Este respaldo se ampliará si su petición tiene presencia mediática sostenida en mensajes elaborados a partir de los resultados de trabajo de un think tank. El análisis de las demandas sociales en la esfera pública en el marco teórico de la tematización revela el poder que tienen estos centros de estudio para introducir temas, incorporar actores, marcar líneas de argumentación e intervenir en las soluciones políticas. Además, los investigadores cuentan con un acceso privilegiado en los 
medios de comunicación lo que ofrece una participación directa como promotores de demandas sociales para buscar el apoyo de las personas implicadas en la discusión y solución del tema en conflicto.

Sin duda, la tematización es un medio decisivo en la formulación y negociación de los problemas sociales. Los grupos se enfrentan no sólo para definir su demanda, ante la opinión pública, sino para influir en las personas que tomarán las soluciones. Este escenario involucra a un grupo de actores que tienen distintos intereses respecto al tema en disputa, así como recursos desiguales para participar en los medios de comunicación; de ahí que se debe de considerar el trabajo de los think tanks y a sus investigadores para instrumentar estrategias comunicativas que tengan como objetivo promover, reforzar 0 intervenir en la discusión pública de los conflictos sociales.

\section{Bibliografía}

Badia, L. (1992), De la persuasió a la tematizació. Introducció a la comunicació política moderna. Pórtic. Barcelona.

Borrat, H. (1998). Fer Europa. Els periodics, protagonistas del post-Mastrich. Centre de Investigació de la Comunicació. Barcelona.

Castillo, A. (2010), Relaciones públicas y think tanks en América Latina: Estudio sobre su implantación y acción. Revista Razón y Palabra. No.70.

Castillo, A. (2011), Lobby y comunicación. El lobbyng como estrategia comunicativa. Comunicación Social. Sevilla-Zamora.

Grossi, G; (1985). Rapresentanza e representazione. Milano. Franco Angelli.

Gusfield, J. (1981), The cultura of public problems. Chicago University Press.

Luhmann, N. (1978). Offentliche meinung. Alemania.

Luhamnn, N. (1989), Complexitat social i opinion pública. Periodística. Núm 1, Barcelona.

Marletti, G. (1985), Prima e dopo. Tematizzazione e comunicazione política. "UTP" 68. Tori.

Pasquino, Gianfranco (ed) (1987): Mas media e sistema politico. Milano 
Raboy, M. y Bernard D. (eds). (1992), Media, crisis and democracy. Sage. London.

Requejo (1991). Els think tanks en Catalunya. Universidad Pompeu Fabra.

Rositi, F (1882), I modi dell'argumentazioni elópinione pubblica. ERI-RAI. Torino.

Santillán, J. (2007). De la información a la opinión. Modelo para el análisis periodístico de los temas político. Revista Razón y Palabra. Vol. 4. pp.22-35.

Santillán, J (2009). La prensa mexicana ante el cambio político. Dikynson. Madrid.

Saperas, E; (1987). Los efectos cognitivos de la comunicación de masas, Barcelona.

Stone, D. (1996). Capturing de political imaginaron. Think tanks and the Policy process. London.

Thompson, J. (1995). Los media y la modernidad: una teoría de los medios de comunicación. Paidós, Barcelona,

Tello, C. (2009). Guía de los think tanks en España. Fundación Ciudadanía y Valores. España.

Xifra, Jordi (2005). Los "think tank" y "advocacy tank" como actores de la comunicación política. Análisis: Quaderns de comunicació i cultura, No. 32. págs. $73-91$

\footnotetext{
'En este texto se utilizará laboratorio de ideas, centros de investigación, grupos de expertos para referirnos a los think tanks. $Y$ también usamos de manera indistinta las palabras: investigadores, analistas, expertos o especialistas para mencionar a sus integrantes.

ii Está definición de la tematización, entendida como el análisis a profundidad de los temas que centran la discusión política, se puede encontrar en los trabajos del grupo de investigación de italianos relativos al campo de la comunicación política en textos como: Grossi, Giorgio (1987); Il campo de la comunicazione política; II campo de la comunicazione política en Pasquino, Gianfranco (ed) (1987): Mas media e sistema politico. Milano. Francesco Angeli 2a ${ }^{a}$. Edición, Grossi, Gorgio (1985): Representazione e reppresentazione. Milano. Franco Marletti, Giorgio (1985): Prima e dopo. Tematizzazione e comunicazione politica. "UTP" 68. Tori. Eri. Rositi, Franco (1982), I modell'argumentazione e l'opinione publica. ERI-RAI. Torino.

Para citar este artículo

Santillán Buelna, José Ramón (20-06-2012). THINK TANKS Y SUS ESTRATEGIAS COMUNICATIVAS.

REDMARKA - CIECID - Unidad de Investigación en Marketing Aplicado-Universidad de A Coruña Año V, Número 8, V1, pp.201-222ISSN 1852-2300

URL del Documento : cienciared.com.ar/ra/doc.php? $n=1661$

URL de la Revista : cienciared.com.ar/ra/revista.php?wid=39
} 